\title{
Erratum to: Polymorphisms in HIF-1alpha affect presence of lymph node metastasis and can influence tumor size in squamous-cell carcinoma of the glottic larynx
}

\author{
F. Mera-Menéndez • A. Hinojar-Gutiérrez • M. Guijarro Rojas • \\ J. García de Gregorio • E. Mera-Menéndez • J. J. Sánchez • \\ M. Quintanilla $\cdot$ L. Cerezo $\cdot$ C. Gamallo
}

Published online: 2 October 2012

(C) Federación de Sociedades Españolas de Oncología (FESEO) 2012

\section{Erratum to: Clin Transl Oncol DOI}

10.1007/s12094-012-0930-Z

In the original published article, the category and subcategory are given wrong.

It should read: RESEARCH ARTICLE (not EDUCATIONAL SERIES - BLUE SERIES).

The online version of the original article can be found under doi:10.1007/s12094-012-0930-z.

F. Mera-Menéndez · A. Hinojar-Gutiérrez $(\bowtie)$.

J. G. de Gregorio

Departamento de Otorrinolaringología, Hospital Universitario

de La Princesa, Universidad Autónoma de Madrid, 4 a Planta,

C/Diego de Leon, 62, 28006 Madrid, Spain

e-mail: ahinojargut@yahoo.es

\section{Guijarro Rojas · C. Gamallo ( $\square)$}

Departamento de Anatomía Patológica, Hospital Universitario de La Princesa, Universidad Autónoma de Madrid, Planta $2^{\circ}$, C/Diego de Leon, 62, 28006 Madrid, Spain

e-mail: cgamallo.hlpr@salud.madrid.org

E. Mera-Menéndez

Departamento de Sistemas Informáticos y Computación,

Universidad Complutense de Madrid, Madrid, Spain

\section{J. J. Sánchez}

Departamento de Medicina Preventiva, Facultad de Medicina,

Universidad Autónoma de Madrid, Madrid, Spain
M. Quintanilla

Instituto de investigaciones Biomédicas “Alberto Sols", CSIC-UAM, Universidad Autónoma de Madrid,

Madrid, Spain

L. Cerezo $(\square)$

Departamento de Oncología Radioterápica, Hospital

Universitario de la Princesa, Universidad Autónoma de Madrid,

Planta-1, C/Diego de León, 62, 28006 Madrid, Spain

e-mail: 1cerezo.hlpr@salud.madrid.org

C. Gamallo

Centro de Investigación Biomédica en Red de Enfermedades Hepáticas y Digestivas (CIBERHED), Barcelona, Spain 